\title{
Examples of Compact Matrix Pseudogroups Arising from the Twisting Operation
}

\author{
N. Andruskiewitsch ${ }^{\star}$ and B. Enriquez \\ Centre de Mathématiques, École Polytechnique, F-91128 Palaiseau, France \\ Received June 10, 1991; in revised form March 13,1992
}

\begin{abstract}
We construct multiparameter compact matrix pseudogroups of all types and show their representation theories are the same as their classical analogs.
\end{abstract}

\section{Introduction}

This paper is concerned with the problem of constructing families of multiparameter quantum groups. This problem was first raised in [M1] and subsequently treated in [T, Re1, OY, H, LS].

In [Dr3], Drinfeld gave a method to "twist" quasi-Hopf algebra structures. This idea was used by Reshetikhin to give multiparameter examples of quasi-triangular Hopf algebras, at the formal level. He also constructed the corresponding bialgebras (over C) of rational functions on the quantum group (cf. [Re1]). He works with a complex simple Lie algebra with fixed Cartan decomposition; the parameter space for these deformations is the second exterior power of the Cartan subalgebra.

The first purpose of this work is to give complex versions of these Hopf algebras, the parameter $q$ being positive $\neq 1$ (Sect. 4). We also construct the Hopf algebras of rational functions (Sect. 5), using the results of [A].

We then find out the conditions for these algebras to yield compact matrix pseudogroups in the sense of [W1] (CMP) (Sect. 5). The test for that is clear from the Tannakian viewpoint: some representation of the quantized enveloping algebra should bear an invariant inner product. The existence of this product in the twisted case follows from the results of Rosso (in the classical case), the first author (in the classical and $E_{6}, E_{7}$ cases) and Tiraboschi (in the $G_{2}, F_{4}$, and $E_{8}$ ones). We thus obtain non-simply connected versions of the quantum groups considered by Levendorskii and Soibelman. (Note that the existence of an invariant inner product remains implicit in [LS]).

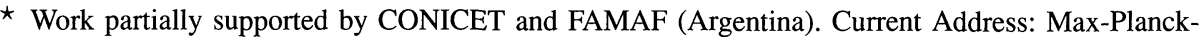
Institut für Mathematik, Gottfried-Claren-Strasse 26, W-5300 Bonn 3, FRG
} 
We study the representation theory of the CMP's, using general arguments within the framework of [A] and the main result of [L2] (Sect. 10).

We would like to thank P. Cartier for interesting discussions.

\section{Conventions}

Let $\mathscr{A}$ be a Hopf algebra over $\mathbf{C}$, with comultiplication $\Delta$, bijective antipode $S$ and counit $\varepsilon$. Let $\varrho: \mathscr{b} \rightarrow$ End $V$ be a finite dimensional representation of $A$, $\varrho^{d}: \mathscr{A} \rightarrow$ End $V^{*}$ be given by $\varrho^{d}=\varrho^{t} S$. If $W$ is a vector space, we shall denote by $\mathscr{T}(W)$ the tensor algebra of $W$. Let $\phi_{\varrho}: \mathscr{T}\left(V \otimes V^{*}\right) \rightarrow \mathscr{A}^{*}$ be induced by $\left\langle\phi_{\varrho}(v \otimes \mu), x\right\rangle=\langle\mu, \varrho(x) v\rangle$; the image of $\phi_{\varrho} \oplus \phi_{\varrho^{d}}: \mathscr{T}\left(\left(V \otimes V^{*}\right) \oplus\left(V \otimes V^{*}\right)\right) \rightarrow \mathscr{A}^{*}$ will be denoted $\operatorname{Coeff}(\varrho)$.

Let $\mathscr{B}$ be another Hopf algebra. A Hopf algebra pairing between $\mathscr{B}$ and $\mathscr{B}$ is a bilinear form $\langle\rangle:, \mathscr{A} \times \mathscr{B} \rightarrow \mathbf{C}$ which transforms operations into co-operations. That is, for example, $\left\langle\Delta(a), b \otimes b^{\prime}\right\rangle=\left\langle a, b b^{\prime}\right\rangle,\langle S(a), b\rangle=\langle a, S(b)\rangle$, etc. If in addition the pairing induces a monomorphism $\mathscr{B} \rightarrow \mathscr{b}^{*}$, then we shall say that $\mathscr{B}$ is dual to $\mathscr{A}$.

Let $T$ be an antilinear multiplicative involution of $\mathscr{b}$. Let $\alpha \mapsto \alpha^{*}$ be the automorphism of $\mathscr{b}^{*}$ given by $\left\langle\alpha^{*}, x\right\rangle=\overline{\langle\alpha, T(x)\rangle}$.

We shall fix a Cartan matrix $A=\left(a_{i j}\right) \in \mathbf{Z}^{n \times n}$ and a diagonal matrix $D=\left(d_{1}, \ldots, d_{n}\right), d_{i} \in \mathbf{Z}$, such that $D A=A D$; i.e. $d_{i} a_{i j}=d_{j} a_{j i}$, for all $i, j$.

\section{The Twisted Compact Matrix Pseudogroups}

\section{A General Construction of CMP's}

We recall from [A] how to construct CMP's from Hopf-algebraic data ( $\tau$ will denote the transposition of the tensor product of two vector spaces):

Proposition. i) Let us assume that there exists an isomorphism of vector spaces $M: V \rightarrow V$ such that $M(a v)=S^{2}(a) M(v)$ for all $a \in \mathcal{A}, v \in V$. Then $\operatorname{Coeff}(\varrho)$ is a Hopf algebra dual to $\mathscr{A}$ and $(V, \varrho)$ is an Coeff $(\varrho)$-comodule.

ii) Let us assume that

$$
\Delta \circ T=(T \otimes T) \circ \tau \circ \Delta \text {. }
$$

Then $\left(\mathscr{b}^{*}, *\right)$ is a *-algebra. Moreover, let $\mathscr{B} \subset \mathscr{A}^{*}$ be a $*$-stable Hopf algebra dual to $\mathscr{A}$ and suppose that

$$
S T S=T
$$

Then $\mathscr{B}$ is a*-Hopf algebra.

iii) Assume that in addition there exists an antilinear isomorphism $J: V \rightarrow V^{*}$ such that

$$
J(x v)=T(x) J(v), \quad x \in \mathscr{b}, v \in V .
$$

Let $\left(\mathbf{u}_{i j}\right)=\left(\phi_{\varrho}\left(v_{j} \otimes \mu_{i}\right)\right) \in \operatorname{Coeff}(\varrho)^{\operatorname{dim} V \times \operatorname{dim} V}$. Then $\operatorname{Coeff}(\varrho)$ is a $*$-Hopf algebra generated by the entries of $\mathbf{u}$.

iv) Let $\varrho, T, J$ satisfy the hypothesis above. Let (|):V $\times V \rightarrow \mathbf{C}$ be the sesquilinear form given by $(v \mid w)=J(w)(v)$. Assume that

(|) is an inner product, and $M$ is symmetric positive for it . 
Then Coeff $(\varrho)$ admits a norm \|\| such that $\left\|x x^{*}\right\|=\|x\|\left\|x^{*}\right\|$. Let us denote $C^{*}(\varrho)$ the completion of $\operatorname{Coeff}(\varrho)$ with respect to \|\| . Then $\left(C^{*}(\varrho), \mathbf{u}\right)$ is a CMP.

Remarks. a) The condition (3) is equivalent to $(x v \mid w)=\left(v \mid T S^{-1}(x) w\right)$, for all $x \in \mathscr{A}, v, w \in V$. Let $*: \mathscr{b} \rightarrow \mathscr{C}$ be the application $x \mapsto T S^{-1}(x)$. Then $(\mathscr{b}, *)$ is a $*$-algebra and (3) means the classical definition of a unitary representation of a $*$-algebra. Moreover, the $*$-operation in $\mathscr{f}^{*}$ is given by $\overline{\left\langle\alpha^{*}, x\right\rangle=\left\langle\alpha,(S(x))^{*}\right\rangle}$, compare with [So].

b) If $\mathscr{b}$ is quasitriangular, then the hypothesis of Proposition 1 i) is fulfilled, see [Dr2].

c) As $\hat{\mathscr{b}}$ denotes the set of classes of irreducible representations of $\mathscr{A}$, the set of classes of irreducible finite dimensional corepresentations of $C^{*}(\varrho)$ will be denoted $C^{*}(\varrho)^{\vee}$.

d) Examples of this construction with the help of quantized enveloping algebras are now well-known.

e) P. Cartier pointed out that the proof in [A] was not complete: there the continuity of $\Delta$ is not checked. This is easily cured using [W2], Theorem 1.3. Let us note that one advantage of $[\mathrm{A}]$ is that it gives a formula for the Haar measure.

\section{The Twisting Operation}

Now we shall recall how to twist the coalgebra structure, see [Re1, Dr3]:

Proposition. Let $(\mathscr{A}, \Delta, S, \varepsilon)$ be a Hopf algebra. Let $F=\sum f_{i} \otimes f^{i} \in \mathscr{A} \otimes \mathscr{A}$ invertible satisfying

$$
\begin{aligned}
F_{23}(1 \otimes \Delta) F & =F_{12}(\Delta \otimes 1) F, \\
(\varepsilon \otimes 1) F & =(1 \otimes \varepsilon) F=1 .
\end{aligned}
$$

Let $u=\sum f_{i} S\left(f^{i}\right)$. Then $u$ is invertible. Put $\Delta^{F}(a)=F \Delta(a) F^{-1}, S^{F}(a)=$ $u S(a) u^{-1}$.

Then $\left(\mathscr{b}, \Delta^{F}, S^{F}, \varepsilon\right)$ is a Hopf algebra, denoted $\mathscr{A}^{F}$. (Note that the algebra structure does not change.)

A similar statement holds in the case of topological Hopf algebras. We briefly recall Reshetikhin's construction. Let $\mathbf{g}$ be a simple complex Lie algebra with fixed Cartan decomposition; let $\mathscr{A}=U_{h} \mathbf{g}$ be the Drinfeld quantized enveloping algebra [over $\mathbf{C}((h))$ ] associated to it. Let $f$ be an antisymmetric tensor in $\mathbf{h} \otimes \mathbf{h}$, where $\mathbf{h}$ denotes the Cartan algebra of g. Then $F=\exp (h f)$ satisfies (5), (6).

Let us now shortly discuss how the twisting of the Hopf algebra alters the construction of the coefficient algebra and the related CMP. Let us, however, remark that the following is mostly useful at the formal level, and that our argument doesn't repose on it. It were, however, these conditions (with $\psi=1$ ) that led us to the restrictions on the parameters we needed (Corollary 5).

First, if $M$ intertwines $\varrho$ and $\varrho S^{2}$, then $\varrho(u) M$ intertwines $\varrho$ and $\varrho\left(S^{F}\right)^{2}$.

Lemma. i) Assume that $T$ satisfies (1), with respect to $\Delta$. Let $\psi$ be an invertible element of $\mathscr{A}$. If

$$
(\psi \otimes \psi)(T \otimes T) \tau(F)=F \Delta(\psi),
$$

then $T^{\psi}:=\psi T \psi^{-1}$ satisfies (1), with respect to $\Delta^{F}$, and $\mathscr{A}^{*}$ (with the twisted multiplication) is a *-algebra. 
Moreover, if $\mathscr{B} \subset \mathscr{b}^{*}$ is a *-stable Hopf algebra dual to $\mathscr{C}$ and $T$ satisfies (2), then $\mathscr{B}$ is a*-Hopf algebra.

ii) Assume that in addition there exists an antilinear isomorphism $J: V \rightarrow V^{*}$ such that $J(x v)=T(x) J(v), x \in \mathscr{A}, v \in V$. Then $J^{\psi}=\psi J=J T(\psi): V \rightarrow V^{*}$ is an antilinear isomorphism and satisfies $J^{\psi}(x v)=T^{\psi}(x) J^{\psi}(v)$, for all $x \in \mathscr{b}, v \in V$. In particular, $\operatorname{Coeff}(\varrho)^{F}$ is a *-Hopf algebra.

iii) Let $\varrho, T, J$ satisfy the hypothesis above. Let $(\mid): V \times V \rightarrow \mathbf{C}$ be the sesquilinear form given by $(v \mid w)=J(w)(v)$. Assume that $(\mid)$ is an inner product. Let $(\mid)^{\psi}$ be defined by $(v \mid w)^{\psi}=J^{\psi}(w)(v)$. Assume that $T S^{-1} \psi=\psi$ and $(T(\psi) v \mid v)>0$. Then the completion of $\operatorname{Coeff}(\varrho)^{F}$ with respect to a suitable norm is a compact matrix pseudogroup.

Remarks. 1) If $F$ satisfies (6) but not (5), then the above construction gives a quasiHopf algebra, see [Dr3]. Equation (5) is a cocycle condition on $F$.

2) The non-triviality of the twisting (that is, the fact it is not the result of an inner automorphism) is ensured when $F$ is not a coboundary, i.e. of the form $(u \otimes u) \Delta u^{-1}$. This can be checked in the case of [Re1] (at the formal level) developing $u$ in powers of $h$ and checking that no $u_{1} \in U \mathbf{g}$ can verify $\Delta u_{1}-\left(1 \otimes u_{1}+u_{1} \otimes 1\right)=$ $f_{\alpha \beta}\left(H^{\alpha} \otimes H^{\beta}-H^{\beta} \otimes H^{\alpha}\right)$ (because the coproduct in $U \mathbf{g}$ is cocommutative).

\section{Twisting an Action on an Algebra}

Now let $\mathscr{C}$ be an associative algebra, with multiplication $m_{\mathscr{C}}$ and unit $1_{\mathscr{C}}$, provided with an action $\sigma$ of $\mathscr{b}$ such that

$$
\begin{aligned}
\sigma(a)(x y) & =m_{\mathscr{C}}((\sigma \otimes \sigma) \Delta(a)(x \otimes y)), \\
\sigma(a)\left(1_{\mathscr{C}}\right) & =\varepsilon(a)\left(1_{\mathscr{C}}\right), \quad a \in \mathscr{B},, x, y \in \mathscr{C} .
\end{aligned}
$$

Let $m_{\mathscr{C}}^{F}(x \otimes y)=m_{\mathscr{C}}\left(F^{-1}(x \otimes y)\right)$.

Proposition. $\left(\mathscr{B}, m_{\mathscr{C}}^{F}\right)$ is an associative $k$-algebra with unit $1_{\mathscr{C}}$, denoted $\mathscr{B}^{F}$, and $\sigma$ defines an action of $\mathscr{C}^{F}$ on $\mathscr{C}^{F}$.

Proof. This follows from (5), (6).

Remark. Theorem 2 in [Re1] follows from this; we shall use a rational version of this "formal" result in Sect. 12.

\section{Definition of a Complex Version of the Twisted Quantum Enveloping Algebra}

Let $F=\left(f_{i j}\right) \in \mathbf{C}^{n \times n}$ be antisymmetric, let $q$ be a positive real number, $q \neq 1$. $U_{q, F}(A)$ is the associative unital $\mathbf{C}$-algebra defined by generators $E_{i}, F_{i}, K_{i}^{s}$, $1 \leq i \leq n, s \in \mathbf{C}\left(K_{i}^{1}\right.$ will be denoted $\left.K_{i}\right)$, and relations

$$
\begin{aligned}
K_{i}^{s} K_{i}^{t} & =K_{i}^{s+t}, \quad K_{i}^{s} K_{j}^{t}=K_{j}^{t} K_{i}^{s}, \quad K_{i}^{0}=1, \\
K_{i}^{s} E_{j} & =q^{d_{i} a_{i j} s} E_{j} K_{i}^{s}, \quad K_{i}^{s} F_{j}=q^{-d_{i} a_{i j} s} F_{j} K_{i}^{s}, \\
E_{i} F_{j}-F_{j} E_{i} & =\delta_{i j} \frac{K_{i}-K_{i}^{-1}}{q^{d_{i}}-q^{-d_{i}}},
\end{aligned}
$$


and if $i \neq f^{\prime} j$

$$
\sum_{h+l=1-a_{i j}}(-1)^{h} E_{i}^{(l)} E_{j} E_{i}^{(h)}=0, \quad \sum_{h+l=1-a_{i j}}(-1)^{h} F_{i}^{(l)} F_{j} F_{i}^{(h)}=0 .
$$

Let us introduce $\Delta_{F}, S$, and $\varepsilon$ by

$$
\begin{aligned}
& \Delta_{F}\left(E_{i}\right)=E_{i} \otimes\left(\prod_{\alpha \beta} K_{\alpha}^{d_{i} a_{i \beta} f_{\beta \alpha}}\right)+K_{i}\left(\prod_{\alpha \beta} K_{\alpha}^{d_{i} a_{i \beta} f_{\alpha \beta}}\right) \otimes E_{i}, \\
& \Delta_{F}\left(F_{i}\right)=F_{i} \otimes K_{i}^{-1}\left(\prod_{\alpha \beta} K_{\alpha}^{-d_{i} a_{i \beta} f_{\beta \alpha}}\right)+\left(\prod_{\alpha \beta} K_{\alpha}^{-d_{i} a_{i \beta} f_{\alpha \beta}}\right) \otimes F_{i}, \\
& \Delta_{F} K_{i}^{s}=K_{i}^{s} \otimes K_{i}^{s} \\
& S\left(E_{i}\right)=-K_{i}^{-1} E_{i}, \quad S\left(F_{i}\right)=-F_{i} K_{i}, \quad S\left(K_{i}^{s}\right)=K_{i}^{-s}, \\
& \varepsilon\left(E_{i}\right)=0, \quad \varepsilon\left(F_{i}\right)=0, \quad \varepsilon\left(K_{i}^{s}\right)=1 .
\end{aligned}
$$

Note that

$$
S^{-1}\left(E_{i}\right)=-E_{i} K_{i}^{-1}, \quad S^{-1}\left(F_{i}\right)=-K_{i} F_{i} \quad S^{-1}\left(K_{i}^{s}\right)=K_{i}^{-s} .
$$

Lemma. $\Delta_{F}$ (respectively $S, \varepsilon$ ) extend on $U_{q, F}(A)$ to an algebra morphism to $U_{q, F}(A) \otimes_{\mathrm{C}} U_{q, F}(A)$ (respectively to $\left.U_{q, F}(A)^{o p}, \mathbf{C}\right)$. These operations make $U_{q, F}(A)$ into a Hopf algebra.

Proof. It can be deduced from the considerations in the preceding section; but it is straightforward to check directly all the axioms. The necessity of the antisymmetry of $F$ appears when checking the axiom of the antipode.

Remark. This algebra is slightly different from that presented in [OY]; in that work, the parameter $q$ may be a complex number, different from a root of unity.

\section{Construction of the $\mathrm{CMP}$}

In what follows, we shall fix $q, A, F$ and write $U$ instead of $U_{q, F}(A)$.

Let $U_{\text {disc }}$ be the subalgebra of $U$ spanned by $E_{i}, F_{i}, K_{i}^{ \pm 1}$; it is clearly isomorphic to the quantized universal enveloping algebra considered in [L1]. Let $\mathbf{m}=$ $\left(m_{1}, \ldots, m_{n}\right) \in \mathbf{Z}^{n}$. For any $U_{\text {disc }}$-module $V$, let $V^{\mathbf{m}}$ be the weight space corresponding to $\mathbf{m}$; i.e. $V^{\mathbf{m}}=\left\{v \in V: K_{i} v=q^{d_{i} m_{i}} v \forall i\right\}$, see [L1]. Let $\varrho_{\text {disc }}: U_{\text {disc }} \rightarrow \operatorname{End}(V)$ be a finite dimensional representation such that $V=\bigoplus_{\mathbf{m} \in \mathbf{Z}^{n}} V^{\mathbf{m}}$; it extends to a representation $\varrho$ of $U$ by setting $\varrho\left(K_{i}^{s}\right) v=q^{s d_{i} m_{i}} v$, for any $v \in V^{\mathbf{m}}$. The algebra Coeff $(\varrho)$ is a Hopf algebra after [A] and can be interpreted as the ring of rational functions on a multiparameter quantum group (see also $[\mathrm{H}]$ ).

Let us consider the antilinear multiplicative involution $T$ of $U$ given by

$$
T\left(E_{i}\right)=-F_{i}, \quad T\left(F_{i}\right)=-E_{\imath}, \quad T\left(K_{i}^{s}\right)=K_{i}^{-\bar{s}} .
$$

Clearly $T$ satisfies (2). 
Proposition. If the entries of $F$ belong to $\sqrt{-1} \mathbf{R}$, then $T$ satisfies (1) and any sesquilinear $U_{\text {disc }}$-invariant form on $V$ is $U$-invariant.

Proof. Let us check (1) for $E_{i}$. On the one hand,

$$
\begin{aligned}
\Delta_{F} T\left(E_{i}\right)= & -\Delta_{F}\left(F_{i}\right)=-\left(F_{i} \otimes K_{i}^{-1}\left(\prod_{\alpha \beta} K_{\alpha}^{-d_{i} a_{i \beta} f_{\beta \alpha}}\right)\right. \\
& \left.+\left(\prod_{\alpha \beta} K_{\alpha}^{-d_{i} a_{i \beta} f_{\alpha \beta}}\right) \otimes F_{i}\right) .
\end{aligned}
$$

On the other hand,

$$
\begin{aligned}
(T \otimes T)\left(\tau \Delta_{F}\left(E_{i}\right)\right)= & (T \otimes T)\left(\left(\prod_{\alpha \beta} K_{\alpha}^{d_{i} a_{i \beta} f_{\beta \alpha}}\right) \otimes E_{i}\right. \\
& \left.+E_{i} \otimes K_{i}\left(\prod_{\alpha \beta} K_{\alpha}^{d_{i} a_{i \beta} f_{\alpha \beta}}\right)\right) \\
= & -\left(\left(\prod_{\alpha \beta} K_{\alpha}^{-d_{i} a_{i \beta} \overline{f_{\beta \alpha}}}\right) \otimes F_{i}\right. \\
& \left.+F_{i} \otimes K_{i}^{-1}\left(\prod_{\alpha \beta} K_{\alpha}^{-d_{i} a_{i \beta} \overline{f_{\alpha \beta}}}\right)\right)
\end{aligned}
$$

as claimed. The proof for $F_{i}$ is similar and for $K_{i}$ is trivial. (2) is easy and the first statement is proved. Now let $(\mid)$ be a sesquilinear $U_{\text {disc }}$-invariant form on $V$. Let $v, w \in V^{\mathbf{m}}, s \in \mathbf{C}$. Then $\left(\varrho\left(K_{i}^{s}\right) v \mid w\right)=q^{s d_{i} m_{i}}(v \mid w)=\left(v, \varrho\left(K_{i}^{-\bar{s}}\right) w\right)$ and we are done.

Corollary. Let $A$ be a Cartan matrix and $n$ be its rank. Then to any real positive parameter $q \neq 1$, to any $\mathbf{m} \in \mathbf{Z}^{n}$ and to any $n \times n$ antisymmetric matrix $F$ with purely imaginary entries, it is possible to associate a CMP, by the procedure described in Proposition 1, considering the representation of $U_{q, F}(A)$ induced from the representation of $U_{\text {disc }}$ of highest weight $\left(q^{d_{1} m_{1}}, \ldots, q^{d_{n} m_{n}}\right)$.

Proof. Any finite dimensional representation of a simple Lie algebra of classical, $E_{6}$ or $E_{7}$ type is contained in a tensor product of minuscule representations, and thus has an $U_{q}(A)$-invariant inner product ([A], see also [Ro2] for the classical cases). The existence of an invariant inner product in a quasi-minuscule representation was established by Tiraboschi; this is the case for the 7-dimensional representation of the quantized enveloping algebra of $G_{2}$, the Jordan algebra representation of $F_{4}$ and the adjoint representation of $E_{8}$ (see [Ti]).

Hence, under the restriction of the proposition above on $F$, the extensions to $U_{q, F}(A)$ of these finite dimensional representations admit an invariant inner product, thus fulfilling the hypotheses in Proposition 1.

\section{The Real Forms}

One needs no extra effort to construct, at the $*$-Hopf algebraic level, real forms of the algebras $U_{q, F}(A)$ and $\operatorname{Coeff}(\varrho)$. Let us introduce (cf. [A, 2.4] and [FRT]), the following antilinear multiplicative involutions of $U: T_{j}(1 \leq j \leq n)$ given by

$$
T_{j}\left(E_{j}\right)=(-1)^{\delta_{i j}+1} F_{i}, \quad T_{j}\left(F_{i}\right)=(-1)^{\delta_{i j}+1} E_{i}, \quad T_{j}\left(K_{i}^{s}\right)=K_{i}^{+\bar{s}} ;
$$


$T_{\theta}$ (where' $\theta$ is a diagram automorphism of order two) given by

$$
T_{\theta}\left(E_{i}\right)=-F_{\theta(i)}, \quad T_{\theta}\left(F_{i}\right)=-E_{\theta(i)}, \quad T_{\theta}\left(K_{i}^{s}\right)=K_{\theta(i)}^{-\bar{s}}
$$

$T_{\theta, j}=T_{\theta} T_{j}$, if $\theta$ is as above and $j$ is fixed by $\theta$. (Recall that a diagram automorphism is an application $\theta:\{1, \ldots, n\} \rightarrow\{1, \ldots, n\}$ such that $a_{i j}=a_{\theta(i) \theta(j)}$, for all $i, j$. It extends to a linear automorphism of $\mathbf{C}^{n \times n}$, also called $\theta$.)

It is easy to see that Proposition 5 holds for $T_{j}$; and if $F$ is in addition $\theta$-invariant, also for $T_{\theta}$ and $T_{\theta, j}$.

\section{The Representation Theory}

Let $\mathscr{A}, \varrho, T$, be as in Sect. 0 and assume that they satisfy the hypothesis in Proposition 1 . The purpose of the following sections is to deduce the (finite dimensional) representation theory of the CMP $C^{*}(\varrho)$ from (part of) the representation theory of $\mathscr{A}$. This completes the study of [A]. Then we will specialize to the example presented above.

\section{The Peter-Weyl Theorem}

First we shall recall some facts from [W1]. Let $V$ be a finite dimensional complex vector space. A corepresentation of $C^{*}(\varrho)$ on $V$ is an element $v=\sum t_{i} \otimes x_{i} \in$ $\operatorname{End}(V) \otimes C^{*}(\varrho)$ such that $v \ominus v:=\sum t_{i} t_{j} \otimes x_{i} \otimes x_{j}$ is equal to (id $\left.\otimes \Delta\right) v$. Let $C^{*}(\varrho)^{v}$ be the subspace of $C^{*}(\varrho)$ spanned by the $x_{i}$ such that $t_{i} \neq 0$ (it is the space of the matrix coefficients of this corepresentation).

Fix an inner product on $V$. A corepresentation is called unitary (respectively smooth) if $v$ is unitary [respectively $v \in \operatorname{End}(V) \otimes \operatorname{Coeff}(\varrho)$ ]. A morphism between two corepresentations $(V, v)$ and $\left(V^{\prime}, v^{\prime}\right)$ is an application $t: V \rightarrow V^{\prime}$ such that $(t \otimes \mathrm{id}) v=v^{\prime}(t \otimes \mathrm{id})$. A subspace of $V$ is invariant when it admits a corepresentation such that the inclusion is a morphism. It is clear what $\operatorname{Coeff}(\varrho)^{v}$ means for a smooth $v$.

A corepresentation is called irreducible (respectively completely reducible) if it has no proper invariant subspace (equivalently, the only morphisms from $V$ to $V$ are the multiples of the identity) (respectively is a direct sum of irreducible invariant subspaces).

The category of corepresentations is closed by (finite) direct sums, (finite) tensor products [because the comultiplication is defined on $C^{*}(\varrho)$ ]; the subcategory of smooth corepresentations is in addition closed by the contragredient operation [recall that the antipode is defined only on $\operatorname{Coeff}(\varrho)$ ]. Let $\mathscr{R}$ be the smallest full subcategory of the category of smooth corepresentations containing $\mathbf{u}, \mathbf{u}^{*}$ and closed by direct sums, tensor products and taking the contragredient. Let $C^{*}(\varrho)^{\vee}$ be the set of isomorphy classes of irreducible corepresentations occurring in $\mathscr{R}$. We shall often identify $\alpha \in C^{*}(\varrho)^{\vee}$ with a representant. We close this section recalling the following fundamental facts:

Theorem (see [W1, 4.5, 4.7]). i) Any smooth corepresentation is completely reducible. 
(ii)

$$
\operatorname{Coeff}(\varrho)=\bigoplus_{\alpha \in C^{*}(\varrho)^{\vee}} \operatorname{Coeff}(\varrho)^{\alpha} .
$$

iii) More precisely, let $\left(V_{\alpha}, v_{\alpha}\right)$ be an irreducible corepresentation of type $\alpha,\left\{w_{i}\right\}$ a basis of $V_{\alpha},\left\{w^{i}\right\} \subseteq V_{\alpha}^{*}$ its dual basis, $u(\alpha)_{j}^{i} \in \operatorname{Coeff}(\varrho)$ such that $v=$ $\sum_{i, j} w^{i} \otimes w_{j} \otimes u(\alpha)_{j}^{i}$. Then $\left\{u(\alpha)_{j}^{i}\right\}$ is a basis of $\operatorname{Coeff}(\varrho)$.

\section{From Comodules to Modules}

In this paragraph, $\mathscr{A}$ and $\mathscr{B}$ are arbitrary Hopf algebras, $\mathscr{B}$ dual to $\mathscr{b}$. Recall that a $\mathscr{B}$-comodule is a pair $(M, \mu)$, where $M$ is a complex vector space and $\mu$ is a linear application $M \rightarrow M \otimes \mathscr{B}$ such that (id $\otimes \Delta) \mu=(\mu \otimes$ id) $\mu$ and (id $\otimes \varepsilon) \mu=$ id. There is a well-known equivalence between finite dimensional comodules and corepresentations; namely, if $v=\sum t_{i} \otimes x_{i} \in \operatorname{End}(V) \otimes B$ is a corepresentation, then we define $\mu(m)=\sum t_{i}(m) \otimes x_{i}$. A morphism of comodules is an application $L: M \rightarrow M^{\prime}$ such that $\mu^{\prime} L=(L \otimes \mathrm{id}) \mu$. It is clear how to define direct sums and (thanks to the multiplication) finite tensor products of comodules. Moreover, the antipode enables to consider the contragredient comodule of a finite dimensional one. The following may be found e.g. in [Sw]:

Lemma. Let $(M, \mu)$ be any $\mathscr{B}$-comodule. The application $\mathscr{b} \otimes M \rightarrow M$ given by the following commutative diagram

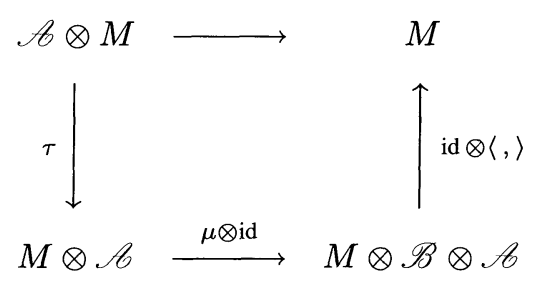

defines a (left) $\mathscr{b}$-module structure on $M$. It gives rise to a functor ८ from the category of right-ஜ̈-comodules to the category of left- $\mathscr{b}$-modules which preserves direct sums, tensor products and (for finite dimensional objects) passing to the contragredient. A comodule $M$ is irreducible if and only if $\iota(M)$ is.

\section{The Representation Theory of CMP's: General Facts}

Now let us recall some facts from [A]. We come back to the notation in Sect. 0. Let us consider $\mathscr{T}\left(\left(V \otimes V^{*}\right) \oplus\left(V^{*} \otimes V\right)\right)$ (respectively $\left.\mathscr{A}^{*}\right)$ as a $\mathscr{A}$-module via the representation induced by $\varrho \otimes$ id $\oplus \varrho^{*} \otimes$ id (respectively the transpose of the right representation, i.e. $\langle x \alpha, y\rangle=\langle\alpha, y x\rangle)$. Then $\phi_{\varrho} \oplus \phi_{\varrho^{d}}$ is a morphism of $\mathscr{A}$-modules and hence $\operatorname{Coeff}(\varrho)$ is a $\mathscr{A}$-module.

Moreover, the hypothesis (4) implies that $\operatorname{Coeff}(\varrho)$ is completely reducible as an Ab-module. That is,

$$
\operatorname{Coeff}(\varrho)=\bigoplus_{\pi \in \hat{\mathscr{b}}} \operatorname{Coeff}(\varrho)_{\pi},
$$


where $\hat{\mathscr{C}}$ is the set of isomorphy classes of irreducible finite dimensional representations of $\mathscr{A}$ and for $\mathscr{\mathscr { C }}$ an $\mathscr{A}$-module and $\pi \in \hat{\mathscr{C}}, \mathscr{C}_{\pi}$ denotes the isotypic component of $\mathscr{U}$ of type $\pi$.

On the other hand, it is always true that $\mathscr{b}_{\pi}^{*}=\phi_{\pi}\left(\pi \otimes \pi^{*}\right.$ ). (Here we are confusing $\pi$ with one representant.) In particular, we have $\operatorname{Coeff}(\varrho)_{\pi} \subset \phi_{\pi}\left(\pi \otimes \pi^{*}\right)$.

Lemma. i) The comultiplication $\Delta: \operatorname{Coeff}(\varrho) \rightarrow \operatorname{Coeff}(\varrho) \otimes \operatorname{Coeff}(\varrho)$ gives rise to a $\operatorname{Coeff}(\varrho)$-comodule structure in $\operatorname{Coeff}(\varrho)$. The Ab-module structure in $\iota(\operatorname{Coeff}(\varrho))$ coincides with the transpose of the right representation.

ii) For any irreducible smooth corepresentation $\alpha, \operatorname{Coeff}(\varrho)^{\alpha}$ is a subcomodule of $\operatorname{Coeff}(\varrho)$.

iii) Let, as in ii), $\alpha \in C^{*}(\varrho)^{\vee}$. Then $\operatorname{Coeff}(\varrho)^{\alpha} \subset \operatorname{Coeff}(\varrho)_{\iota(\alpha)}$.

Proof. i) The first statement follows from the axioms of the comultiplication. Let $x, y \in \mathscr{b}, f \in \operatorname{Coeff}(\varrho)$. Let us write $\Delta(f)=\sum f_{i} \otimes f^{i}$. The action of $\mathscr{b}$ on $\operatorname{Coeff}(\varrho)$ induced by duality is $x f=\sum\left\langle f^{i}, x\right\rangle f_{i}$, and hence $\langle x f, y\rangle=\sum\left\langle f^{i}, x\right\rangle\left\langle f_{i}, y\right\rangle=$ $\langle\Delta(f), y \otimes x\rangle=\langle f, y x\rangle$, i.e. the transposed right action, which is the claim.

ii) follows from the definition of corepresentation.

iii) Let $(V, v)$ be an irreducible smooth corepresentation of type $\alpha,\left\{w_{i}\right\}$ a basis of $V,\left\{w^{\imath}\right\} \subset V^{*}$ its dual basis, $u_{j}^{i} \in \operatorname{Coeff}(\varrho)$ such that $v=\sum_{i, j} w_{i} \otimes w^{j} \otimes u_{j}^{i}$. We claim that

$$
u_{j}^{i}=\phi_{\iota(\alpha)}\left(w^{i} \otimes w_{j}\right)
$$

That is, we need to check that $\left\langle u_{j}^{i}, y\right\rangle=\left\langle w^{i}, y w_{j}\right\rangle$, for all $y \in \mathscr{C}$. But let us recall what the action in the second equality means: $y w_{j}=\sum_{i}\left\langle u_{j}^{i}, y\right\rangle w_{j}$. (Here we are passing from the corepresentation to the comodule and then to the module.) This proves (21) and that

$$
\operatorname{Coeff}(\varrho)^{\alpha}=\phi_{\iota(\alpha)}\left(V_{\iota(\alpha)} \otimes V_{\iota(\alpha)}^{*}\right)
$$

Thus $\operatorname{Coeff}(\varrho)^{\alpha} \subset \mathscr{A}_{\iota(\alpha)}^{*} \cap \operatorname{Coeff}(\varrho)=\operatorname{Coeff}(\varrho)_{\iota(\alpha)}$ and we are done.

Let $\hat{\mathscr{C}}\left[\varrho, \varrho^{d}\right]$ denote the Grothendieck ring of the representations of $\mathscr{A}$ generated by $\varrho$ and $\varrho^{d}$. Now, combining Theorem 7, Lemma 8, and Lemma 9 we obtain the following result:

Theorem. $\iota$ gives rise to a ring isomorphism between the Grothendieck ring of the smooth corepresentations of $C^{*}(\varrho)$ and $\hat{\mathscr{C}}\left[\varrho, \varrho^{d}\right]$. Furthermore,

$$
\operatorname{Coeff}(\varrho)=\bigoplus_{\alpha \in C^{*}(\varrho)^{\vee}} \operatorname{Coeff}(\varrho)^{\alpha}
$$

is the decomposition of $\operatorname{Coeff}(\varrho)$ in isotypic components with respect to the $\mathscr{A}$ module structure, obtained transposing the right multiplication. In particular, for any irreducible $\pi$ in $\hat{\mathscr{C}}\left[\varrho, \varrho^{d}\right]$, $\operatorname{Coeff}(\varrho)_{\pi}=\phi_{\pi}\left(\pi \otimes \pi^{*}\right)$, and $\operatorname{dim} \operatorname{Coeff}(\varrho)_{\pi}=\operatorname{dim}(\pi)^{2}$.

Proof. Lemma 8 shows that the ring homomorphism is well defined. The injectivity is checked as follows: let $\lambda$ and $\mu$ be two smooth corepresentations of $C^{*}(\varrho)$, such that $\iota(\lambda)$ is isomorphic to $\iota(\mu)$ as $\mathscr{b}$-module. Fix a vector space $V$ carrying both representations and a basis $\left(e_{i}\right)$ of $V$. Express each coaction by $\delta_{\lambda}\left(e_{i}\right)=\sum_{j} b_{i}^{j} \otimes e_{j}$ 
and $\delta_{\mu}\left(e_{i}\right)=\sum_{j} b_{i}^{\prime j} \otimes e_{j}$. Then $\left\langle a, b_{i}^{j}\right\rangle=\left\langle a, b_{i}^{\prime j}\right\rangle$ for each $a$ in $\mathscr{A}$ and $\lambda=\mu$ by duality. Now comparing the decompositions of Theorem 7 and of (20), using the inclusion of Lemma 9 and the injectivity of $\iota$ we get $\operatorname{Coeff}(\varrho)^{\alpha}=\operatorname{Coeff}(\varrho)_{\iota(\alpha)}$ and (22). Once one has shown the surjectivity of $\iota$, the following statements are obvious. Let $\pi$ be an irreducible subrepresentation of $\mathscr{T}\left(\left(V \otimes V^{*}\right) \oplus\left(V^{*} \otimes V\right)\right)$, then $\pi$ is included in some homogeneous component $\mathscr{T}_{n}\left(\left(V \otimes V^{*}\right) \oplus\left(V^{*} \otimes V\right)\right)$. Now, $\mathscr{T}_{n}\left(\left(V \otimes V^{*}\right) \oplus\left(V^{*} \otimes V\right)\right)$ is a $\operatorname{Coeff}(\varrho)$-comodule, that is made into a $\mathscr{C}$-module; but, as we are in the case of finite dimensions, any submodule comes from a subcomodule. Irreducibilities in both cases are equivalent, and we are done.

\section{The Corepresentation Theory of $C^{*}(\varrho)$}

In this paragraph, we shall use the notation of Sect. 5. The last point of this section is to relate the representation theories of $U$ and $U_{\text {disc }}$. Let $\operatorname{Rep}\left(U_{\text {disc }}\right)$ [respectively $\operatorname{Rep}(U)]$ be the category of finite dimensional $U_{\text {disc }}$ (respectively $U$ )-representations such that the action of $K_{i}$ (respectively $K_{i}^{s}$ ) is diagonalizable for all $i$ (respectively for all $i$ and $s$ ) with eigenvalues $q^{d_{i} m}$ (respectively $q^{d_{i} m s}$ ) for some $m \in \mathbf{Z}$. We already constructed a functor $\varrho_{\text {disc }} \mapsto \varrho$ which is clearly an isomorphism and preserves direct sums, subrepresentations, quotients. Now let us consider in $U_{\text {disc }}$ the coalgebra structure given in Sect. 2. Then the above functor also preserves the passage to the contragredient. The only non-trivial point to investigate is the tensor product.

Let $(V, \varrho),(W, \sigma)$ be two such finite dimensional modules, $V=\bigoplus_{\mathbf{m} \in \mathbf{Z}^{n}} V^{\mathbf{m}}$, $W=\bigoplus_{\mathbf{p} \in \mathbf{Z}^{n}} W^{\mathbf{p}}$ the respective weight decompositions. $V \otimes W$ has two $U$-module structures: first, the structure induced from $\left(\varrho_{\text {disc }} \otimes \sigma_{\text {disc }}\right) \Delta$; second, $(\varrho \otimes \sigma) \Delta_{F}$. We need to relate them. This is clear at the formal level, cf. Sect. 1; it is only necessary to intertwine by the image of the element defining the twisting. This element, however, does not exist at the "rational" level, i.e. in $U \otimes U$. But its image in $\operatorname{End}(V \otimes W)$ does make sense.

This motivates the following lemma:

Lemma. Let $\mathscr{F}: V \otimes W \rightarrow V \otimes W$ the application which restricted to $V^{\mathbf{m}} \otimes W^{\mathbf{p}}$ is multiplication by $q^{\sum_{\alpha, \beta} f_{\alpha \beta} d_{\alpha} m_{\alpha} d_{\beta} p_{\beta}}$.Then $\mathscr{F}$ intertwines the representations described above.

Proof. Let us show that

$$
\left(E_{i} \otimes\left(\prod_{\alpha \beta} K_{\alpha}^{d_{i} a_{i \beta} f_{\beta \alpha}}\right)+K_{i}\left(\prod_{\alpha \beta} K_{\alpha}^{d_{i} a_{i \beta} f_{\alpha \beta}}\right) \otimes E_{i}\right) \mathscr{F}=\mathscr{F}\left(E_{i} \otimes 1+K_{i} \otimes E_{i}\right)
$$

It suffices to prove it for $u \in V^{\mathbf{m}}, w \in W^{\mathbf{P}}$. But in this case, the left-hand side is

$$
\begin{aligned}
& \sum^{\sum_{\alpha \beta} f_{\alpha \beta} d_{\alpha} m_{\alpha} d_{\beta} p_{\beta}+\sum_{\alpha \beta} d_{\alpha} p_{\alpha} d_{i} a_{i \beta} f_{\beta \alpha}} E_{i} u \otimes w \\
& \quad+q^{\sum_{\alpha \beta} f_{\alpha \beta} d_{\alpha} m_{\alpha} d_{\beta} p_{\beta}+\sum_{\alpha \beta} d_{\alpha} m_{\alpha} d_{i} a_{i \beta} f_{\alpha \beta}} K_{\imath} u \otimes E_{i} w
\end{aligned}
$$


and the right one is

$$
q^{\sum_{\alpha \beta} f_{\alpha \beta} d_{\alpha}\left(m_{\alpha}+a_{i \alpha}\right) d_{\beta} p_{\beta}} E_{i} u \otimes w+q^{\sum_{\alpha \beta} f_{\alpha \beta} d_{\alpha} m_{\alpha} d_{\beta}\left(p_{\beta}+a_{i \beta}\right)} K_{i} u \otimes E_{i} w .
$$

The proof for $F_{i}$ is similar and for $K_{i}^{s}$ it is obvious.

We can summarize the results of this paragraph:

Proposition. The Grothendieck rings of the categories $\operatorname{Rep}\left(U_{\text {disc }}\right)$ and $\operatorname{Rep}(U)$ are isomorphic.

Combining with Theorem 9 and [L2, Theorem 4.13], we deduce

Theorem. The Grothendieck rings of the categories of smooth representations of the CMP constructed in the Corollary 6 and its classical analog are isomorphic, at least if $q$ is transcendental over $\mathbf{Q}$.

Remark. We are grateful to P. Polo for pointing out to us that in fact Lusztig's argument applies to any $q$ which is not a root of 1 .

\section{Link with Quadratic Algebras and a Computation for sl(3)}

In this section, we want to discuss the relation between our construction (when the Cartan matrix is of type $A_{n}$ ), quadratic algebras [M2] and a general theorem of Woronowicz.

To begin with, let us recall [W2, Theorem 1.4]. Let $N$ be a natural number. Let

$$
\mathbf{E}=\left(E_{i_{1}, \ldots, i_{N}}\right) \in \mathbf{C}^{\left(N^{N}\right)}, \quad 1 \leq i_{j} \leq N .
$$

We refer to [W2] for the definition of the non-degeneracy condition. Let $A$ be the universal $C^{*}$-algebra generated by the $N^{2}$ elements $u_{i j}$, subject to the relations

$$
u^{\otimes N} \mathbf{E}=\mathbf{E}, \quad u^{*} u=u u^{*}=\mathrm{id} .
$$

[Here $u=\left(u_{i j}\right)$.] The above mentioned theorem of Woronowicz says that $(A, u)$ is a CMP, provided that $\mathbf{E}$ satisfies the non-degeneracy condition.

Now let us recall some facts about quadratic algebras. Let $q_{i j} \in \mathbf{C}^{N \times N}$. Let us assume that $q_{i j} q_{j i}=q_{i i}=1$. Let $\mathscr{C}$ be the quotient of $\mathbf{C}\left\langle x_{1}, \ldots, x_{N}\right\rangle$ (the free associative algebra in variables $x_{i}$ ) by the relations

$$
x_{j} x_{i}-q_{i j} x_{i} x_{j}
$$

Clearly, $\mathscr{C}=\bigoplus_{m} \mathscr{C}_{m}$ is a quadratic algebra. Let $\mathscr{C}^{!}$be its quadratic dual (cf. [M2]). Let $\xi_{i}$ be defined by $\left\langle\xi_{i}, x_{j}\right\rangle=\delta_{i j}$. Then it is easy to see that

$$
\mathscr{B}^{!}=\mathbf{C}\left\langle\xi_{i}\right\rangle /\left(\xi_{j} \xi_{i}+q_{i j} \xi_{i} \xi_{j}\right) .
$$

This implies in particular that $\mathscr{C}^{!}$is a quantum grassmanian algebra [M2]; i.e., $\operatorname{dim}\left(\mathscr{C}^{!}\right)_{N}=1$ and the multiplication induces a non-singular pairing $\left(\mathscr{C}^{!}\right)_{j} \otimes$ $\left(\mathscr{C}^{!}\right)_{N-j} \rightarrow\left(\mathscr{C}^{!}\right)_{N}$ for all $j$. In particular, $\left(\mathscr{C}^{!}\right)_{j}=0$ if $j>N$. This enables us to define $\mathbf{E} \in \mathbf{C}^{\left(N^{N}\right)}$ by the equality in $\mathscr{C}^{!}$

$$
\xi_{i_{1}} \ldots \xi_{i_{N}}=E_{i_{1}, \ldots, i_{N}} \xi_{1} \ldots \xi_{N}
$$


It is easy to see that the non-degeneracy condition of [W2] follows from the nonsingularity of the pairings between $\mathscr{O}_{1}^{!}$and $\mathscr{O}_{N-1}^{!}$.

Now let $\mathscr{b}, \mathscr{B}$ be Hopf algebras, $\mathscr{B}$ dual to $\mathscr{C}$, and let $\mu: \mathscr{C} \rightarrow \mathscr{C} \otimes \mathscr{B}$ define a (right) comodule structure. Let us assume that $\mu$ is a morphism of algebras. [Equivalently, the $\mathscr{b}$-module structure explained in Sect. 8 satisfies (7)]. Let us assume in addition that $\mu$ is graded, i.e. that each $\mathscr{B}_{m}$ is a subcomodule and $(\mathscr{C})_{j} \otimes(\mathscr{C})_{i} \rightarrow(\mathscr{C})_{i+j}$ is a morphism of comodules. (Conversely, if $\mathscr{C}_{1}$ is a finite dimensional $\mathscr{B}$-comodule and $\mathscr{B}$ is a subcomodule of $\mathscr{B} \otimes \mathscr{O}_{1}$ then the quadratic algebra generated by $\mathscr{B} 1$ with relations $\mathscr{R}$ has a $\mathscr{B}$-comodule structure given by a morphism of algebras.) From this, it follows easily that $\mathscr{\mathscr { C }} !$ is a graded left $\mathscr{B}$ comodule whose structure morphism is a morphism of algebras. In particular, as remarked in [M2], there exists $D \in \mathscr{B}$ such that

$$
\xi_{1} \ldots \xi_{N} \mapsto D \otimes \xi_{1} \ldots \xi_{N}
$$

Now we shall specialize the preceding general remarks to the case we are interested in. Let $\mathscr{A}=U_{q, F}\left(A_{N-1}\right)$. Let $V$ be a complex vector space with basis $\left(x_{i}\right)_{1 \leq i \leq N}$. Let $c_{i j}=\delta_{i j}-\delta_{i, j-1}$. The application $\varrho: \mathscr{b} \rightarrow \operatorname{End}(V)$ given by

$$
E_{i}\left(x_{j}\right)=\delta_{i, j-1} x_{j-1}, \quad F_{i}\left(x_{j}\right)=\delta_{i j} x_{j+1} \quad K_{i}^{s}\left(x_{j}\right)=q^{c_{i j} s} x_{j}
$$

defines the "natural" representation of $\mathscr{A}$, see [Re2] and Sect. 4. Let

$$
q_{i j}=q^{-1} q^{2\left(f_{i j}-f_{i-1, j}-f_{\imath, j-1}+f_{i-1, j-1}\right)} \text { if } i>j,
$$

and put $q_{i j}=1, q_{j i}=q_{i j}^{-1}$. It is easy to see that the subspace of $V \otimes V$ generated by $x_{i} \otimes x_{j}-q_{i j} x_{j} \otimes x_{i}$ is actually a $\operatorname{Coeff}(\varrho)$-subcomodule. (Compare with [Re2] and Proposition 2.) Let $\mathscr{C}$ be the corresponding quadratic algebra. Let $u_{i j}=\phi_{\varrho}\left(x_{j} \otimes \xi_{i}\right)$. As $\left(\mathscr{B}_{1}^{!}\right)^{\otimes N} \rightarrow\left(\mathscr{B}^{!}\right)_{N}$ is a morphism of comodules, we have

$$
E_{i_{1}, \ldots, i_{N}} D=\sum_{j_{1}, \ldots, j_{N}} u_{i_{1}, j_{1}} \ldots u_{i_{N}, j_{N}} E_{i_{1}, \ldots, \imath_{N}} D .
$$

Now from the representation theory of $\mathscr{A}$ developed in the preceding sections and [L2] it follows that $D=1$. That is, we have the first part of (23). The second follows from [A, Lemma 1.4 and Proposition 2.5], for example. Thus we have an epimorphism from the CMP presented by (23) to $C^{*}(\varrho)$. We think that it is an isomorphism. In the case $F=0$, this was proved in [Ro2, Theorem 7].

Remark. So, the fundamental representation of the CMP attached by Woronowicz's theorem to the datum $\mathbf{E}$ is irreducible. In the case $N=3$, this can be checked directly along the lines of [W2]: indeed, the self intertwiners of it belong to the algebra generated by $T, T^{*}$, where $T_{i j}=\sum_{i_{1} i_{2}} E_{j, i_{1}, i_{2}}^{*}$. But in the case $N=3$, the computation gives

$$
\begin{gathered}
E_{123}=1, \quad E_{312}=q^{-2}, \quad E_{231}=q^{-2}, \\
E_{132}=-q^{-1+2 f_{12}}, \quad E_{321}=-q^{-3+2 f_{12}}, \quad E_{213}=-q^{-1+2 f_{12}} .
\end{gathered}
$$

Now, the non-diagonal terms of $\left(T_{i j)}\right.$ vanish and we get

$$
\begin{gathered}
T_{11}=q^{-2}\left(1+\left|q^{-2+4 f_{12}}\right|\right), \quad T_{22}=q^{-2}\left(q^{-2}+\left|q^{4 f_{12}}\right|\right), \\
T_{33}=q^{-2}\left(1+\mid q^{-2+4 f_{12}}\right)
\end{gathered}
$$

and with our assumptions on $q$ and $f_{12}, T$ is scalar. 


\section{Referencés}

[A] Andruskiewitsch, N.: Some exceptional compact matrix pseudogroups. Bull. Soc. Math. Fr. (to appear)

[BD] Belavin, A.A., Drinfeld, V.G.: On the solutions of the classical Yang-Baxter equations for simple Lie algebras. Funkt. Anal. i Prilozhen, (Russian) 3, 1-19 (1982)

[Dr1] Drinfeld, V.G.: Quantum groups. Proc. of the ICM, Berkeley 798-820 (1986)

[Dr2] Drinfeld, V.G.: On almost cocommutative Hopf algebras. Leningrad Math. J. 1(2), 321-342 (1990)

[Dr3] Drinfeld, V.G.: Quasi-Hopf algebras. Leningrad Math. J. 1(6), 1419-1457 (1990)

[FRT] Fadeev, L.D., Reshetikhin, N.Yu., Takhtajan, L.A.: Quantization of Lie groups and Lie algebras. Algebraic Analysis, Papers dedicated to Professor Mikio Saito on the Occasion of His Sixtieth Birthday, pp. 129-139. New York, London: Academic Press 1988

$[\mathrm{H}] \quad$ Hayashi, T.: Quantum groups and quantum determinants. Preprint

[J] Jimbo, M.: A $q$-difference analogue of $U \mathbf{g}$ and the Yang-Baxter equation. Lett. Math. Phys. 10, 63-69 (1985)

[LS] Levendorskii, S., Soibelman, Ya.S.: Algebra of functions of the compact quantum groups, Schubert cells and quantum Tori. Commun. Math. Phys. 139, 141-170 (1991)

[L1] Lusztig, G.: Introduction to quantized enveloping algebras. Proceedings of the Workshop on Representation theory of reductive Lie groups. (Carlos Paz). Birkhäuser (to appear)

[L2] Lusztig, G.: Quantum deformations of certain simple modules over enveloping algebras. Adv. Math. 70, 237-249 (1988)

[M1] Manin. Y.: Multiparametric quantum deformation of the general linear supergroup. Commun. Math. Phys. 123, 163-175 (1989)

[M2] Manin. Y.: Some remarks on Koszul algebras and quantum groups. Ann. Inst. Fourier 37, 191-205 (1987)

[M3] Manin. Y.: Quantum groups and non-commutative differential geometry. Montréal preprint

[OY] Okado, M., Yamane, H.: $R$-matrices with gauge parameters and multiparameter quantized enveloping algebras. Preprint

[Re1] Reshetikhin, N.: Multiparameter quantum groups and twisted quasitriangular Hopf algebras. Lett. Math. Phys. 20, 331-335 (1990)

[Re2] Reshetikhin, N.: Quantized universal enveloping algebras, the Yang-Baxter equation and invariants of links. I, II. Preprint L.O.M.I

[Ro1] Rosso, M.: Finite dimensional representations of the quantum analog of the enveloping algebra of a complex simple Lie algebra. Commun. Math. Phys. 117, 581-593 (1988)

[Ro2] Rosso, M.: Algèbres enveloppantes quantifiées, groupes quantiques compacts de matrices et calcul différentiel non commutatif. Duke Math. J. 61, 11-39 (1990)

[So] Soibel'man, Ya.S.: The algebra of functions on a compact quantum group, and its representations. Leningrad Math. J. 2(1), 161-178 (1990)

[Sw] Sweedler, M.E.: Hopf algebras. Mathematical Lecture Note Series. New York: Benjamin 1969

[T] Takeuchi, M.: A two parameter quantization of $G L(n)$. Proc. Japan Acad., Ser. A 66, 112-114 (1990)

[Ti] Tiraboschi, A.: Compact quantum groups $G_{2}, F_{4}$, and $E_{8}$. C.R. Acad. Sci. Paris, Série 1313 , 913-918 (1991)

[W1] Woronowicz, S.L.: Compact matrix peudogroups. Commun. Math. Phys. 111, 613-665 (1987)

[W2] Woronowicz, S.L.: Tannaka-Krein duality for compact matrix pseudogroups. Twisted $S U(N)$ groups. Invent. Math. 93, 35-76 (1988) 
